


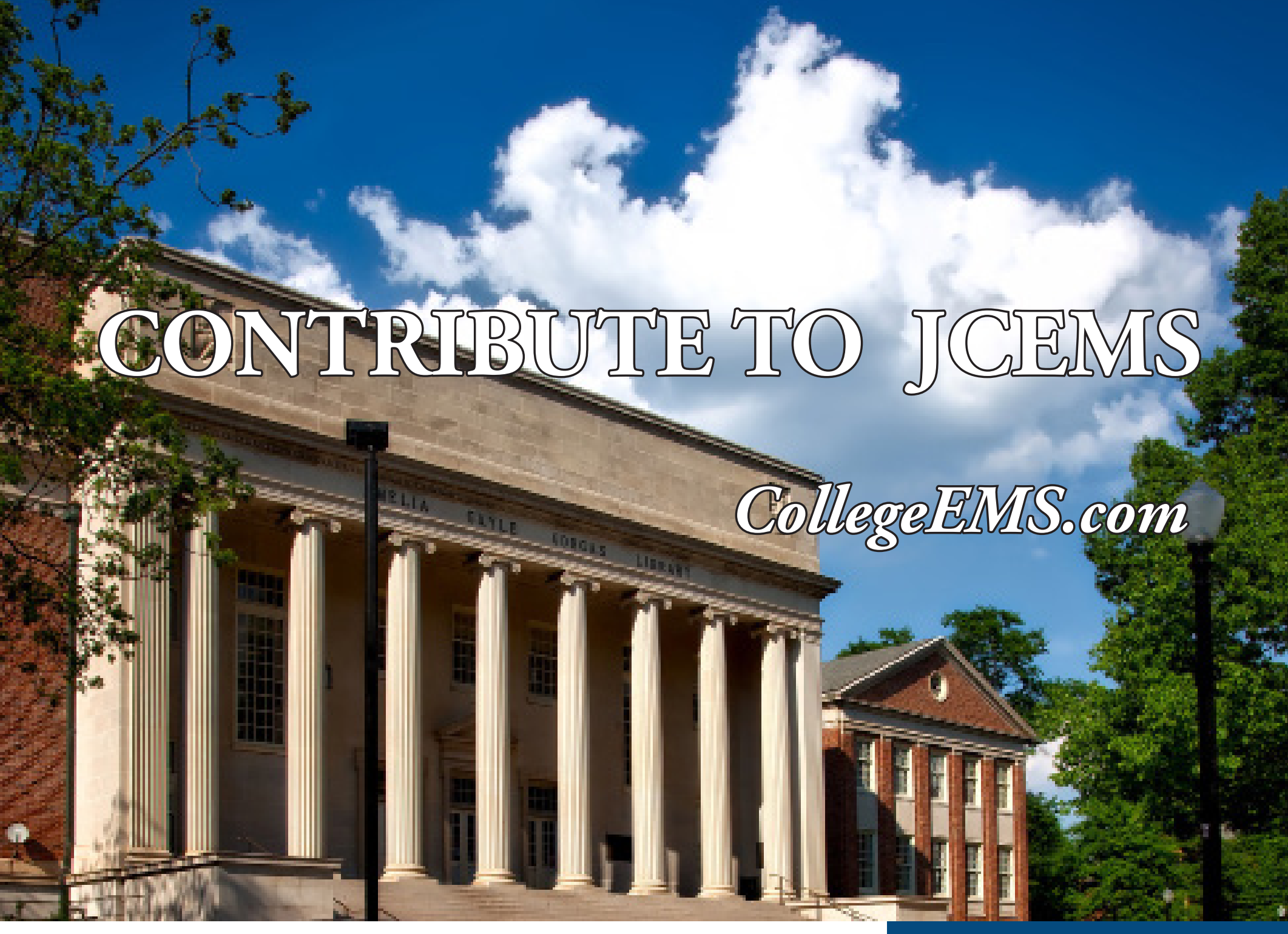

Submit to JCEMS: the official scholarly, peer-reviewed journal of the National Collegiate EMS Foundation.
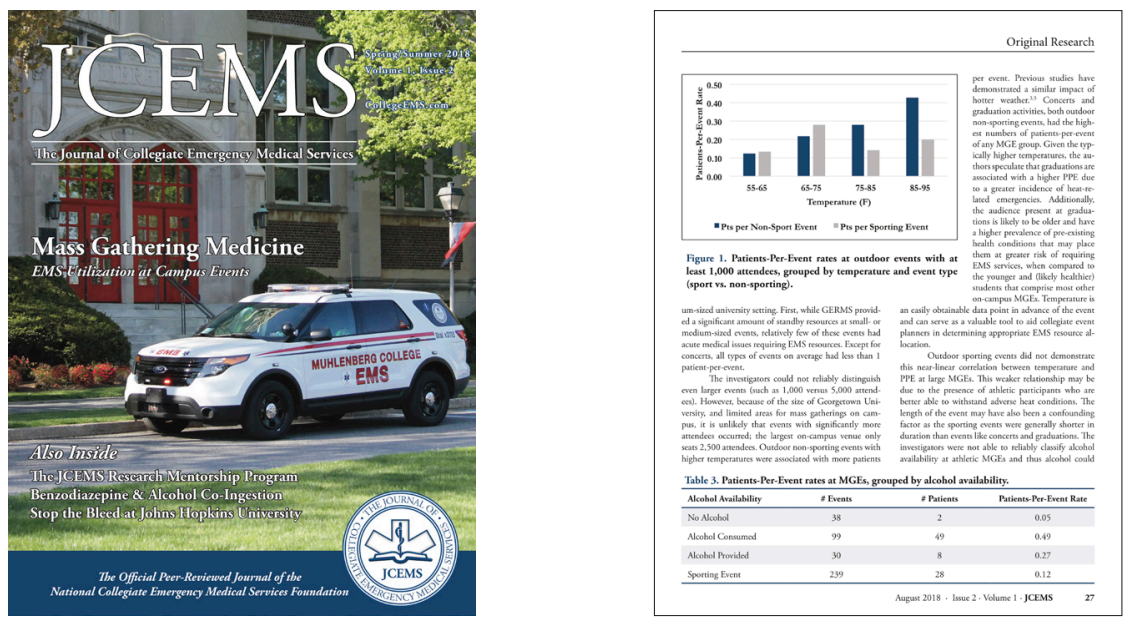

Original research is prioritized. Case reports, reviews, and articles featuring perspectives and commentary are also invited.

\section{Research Mentorship}

We are committed to mentoring student researchers and authors. If you might be interested in conducting a study or submitting a manuscript for publication, consider applying to the JCEMS Research Mentorship Program. Additional details are available at CollegeEMS.com.

\section{Submission Guidelines}

View submission guide at: CollegeEMS.com

Send inquiries to: JCEMS@CollegeEMS.com 


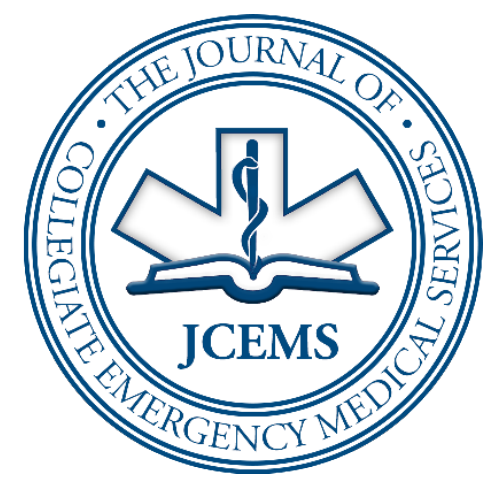

\section{TABLE OF CONTENTS}

\section{The Official Journal of the National Collegiate Emergency Medical Services Foundation}

\section{Editorial}

5 Inside the JCEMS Team

Organizational Structure \&

Opportunities for Involvement

Nicholas M.G. Friedman, BA, EMT \&

Brittany J. Dingler, MHS, PA

\section{News \& Commentary}

7 Interview with Paul Halliday

2019 Collegiate EMS Provider of the Year

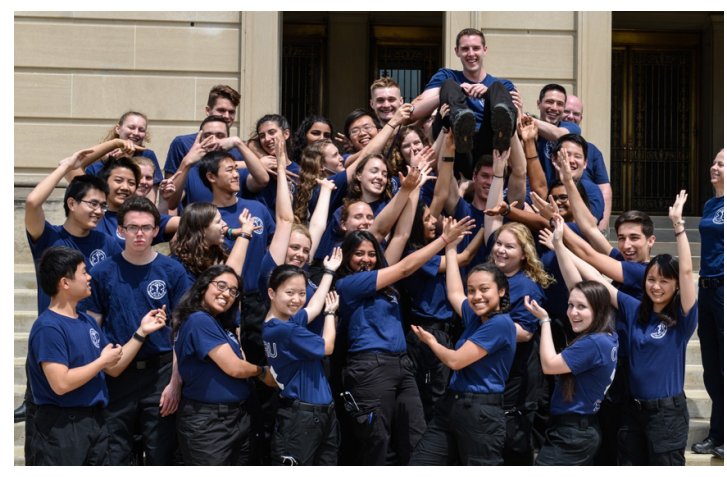

9 Interview with Catherine Gibbs

Winner of the 2019 NCEMSF Vomacka

Student Speaker Competition

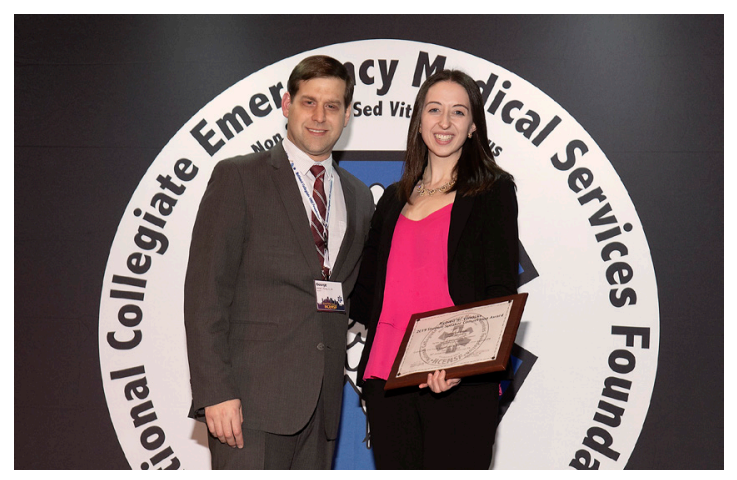

\section{SUPPLEMENTAL}

$2^{\text {th }}$ Annual Conference of the National Collegiate Emergency Medical Services Foundation

Academic Poster Session Abstracts

S1 Development of an Excel-Based Software Solution to Collegiate EMS Shift Scheduling \& Placement
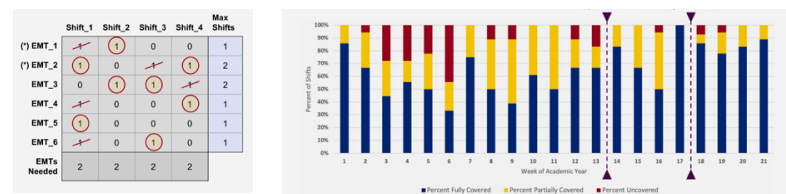

S2 Free of Charge: Analysis of an Urban Collegiate EMS Agency

S3 SIREN: Schema In-Fill Rendered from Entry Notifications

S4 No IV, No Problem: A Cross Sectional Analysis of Antiemetic Therapies in Statewide EMS Protocols

S5 Do EMT students enrolled through a college course perform better in terms of course completion and exam success?

S6 Reducing Barriers to Bystander Intervention: Piloting a Wintersession Program

S7 Integrating Continuing Education Credits to a Collegiate EMS Training Model

\section{Cover Images}

\section{Front Cover:}

Virgina Tech Rescue Squad Command Vehicle

Photo Credit: Virgina Tech Rescue Squad, Virginia Tech

Back Cover:

Piers Park, Boston, MA

Photo Credit: Wasin Pummarin (123RFcom) 


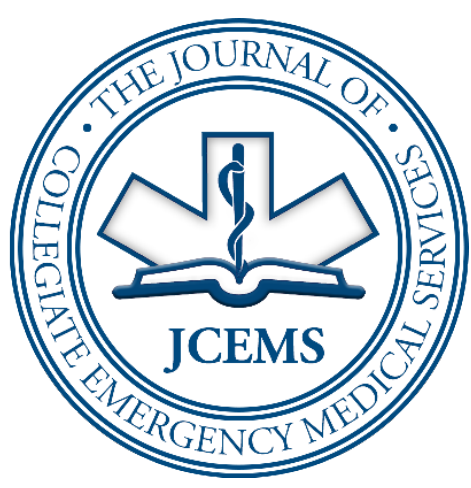

\section{Editors}

Nicholas M.G. Friedman, BA, EMT• Editor-in-Chief

Brittany J. Dingler, MHS, PA • Executive Editor

\section{Editorial Board}

Jose Victor L. Nable, MD, MS, NRP

Georgetown University School of Medicine, Washington, DC

Matthew J. Levy, DO, MS, FACEP

The Johns Hopkins University School of Medicine, Baltimore, MD

Benjamin J. Lawner, DO, MS, EMT-P, FACEP

Temple University School of Medicine, Philadelphia, PA

David Goroff, MS, NRP

New Castle County EMS, New Castle, DE

Albert Jagoda, MD

Skidmore College, Saratoga Springs, NY

Brent Campbell, BA, AEMT-CC

Ambulance Service of Fulton County, Gloversville, NY

Patricia Bosen, MSN, FNP-C

Skidmore College, Saratoga Springs, NY

Joseph M. Grover, MD

University of North Caroline School of Medicine, Chapel Hill, NC

Lauren N. Gorstein, BA, EMT-B

Skidmore College, Saratoga Springs, NY

Michael W. Dailey, MD, FACEP, FAEMS

Albany Medical College, Albany, NY

Jeffrey S. Lubin, MD, MPH, FACEP, FAEMS

Penn State College of Medicine, Hershey, PA

Publishing \& Management

Christopher Gaeta • Associate Manager of Institutional Relations/Business Ops

News \& Outreach

Isabel Anzani • Social Media \& Outreach Editor

\section{Special Programs}

Michael Beautyman • Director of Mentorship Program

Joe Caruso • Associate Manager of Special Events

\section{Reviewers}

JCEMS employs a double-blind peer review process for Original Research, Case Reports, and Reviews. The clinical and scientific quality of this publication relies on the rigorous and diligent reviews provided by independent reviewers with subject-matter expertise. JCEMS is grateful for all anonymous independent reviewers who dedicated their time and expertise to contribute to the collegiate EMS community.

\begin{abstract}
General Information
The Journal of Collegiate Emergency Medical Services (JCEMS) [ISSN 25763687] is the official scholarly, peer-reviewed journal of the National Collegiate Emergency Medical Services Foundation. JCEMS is published by the National Collegiate Emergency Medical Services Foundation.
\end{abstract}

Annual Subscription: Visit https://www.collegeems.com for information on purchasing institutional and personal annual subscriptions.

Reprints: Visit https://www.collegeems.com for information on purchasing reprints and single issues.

Correspondence (Editorial): Address mail to The Journal of Collegiate Emergency Medical Services, National Collegiate Emergency Medical Services Foundation, PO Box 93, West Sand Lake, NY 12196. Email: JCEMS@CollegeEMS.com

Correspondence (Business): Address mail to The Journal of Collegiate Emergency Medical Services, National Collegiate Emergency Medical Services Foundation, PO Box 93, West Sand Lake, NY 12196. Email: JCEMS@CollegeEMS.com

Correspondence (NCEMSF): Address mail to National Collegiate Emergency Medical Services Foundation, PO Box 93, West Sand Lake, NY 12196.

Email: info@ncemsf.org

Copyright: The Journal of Collegiate Emergency Medical Services is an open access publication. Individual authors retain copyright over their own articles. Articles are distributed under the terms of the Creative Commons Attribution 4.0 International (CC BY 4.0) License, which permits unrestricted use, distribution, and reproduction in any medium, provided the original author and source are credited. The full license is available at: https://creativecommons.org/licenses/ by/4.0/. All other content in the journal is copyrighted by the National Collegiate Emergency Medical Services Foundation. All rights reserved.

Online availability: All articles published in print issues of JCEMS are available open access at https://www.collegeems.com. Additional content - not available in print issues - is also published on-line.

Disclaimers: The statements and opinions in articles or other content contained in JCEMS are solely those of the individual authors, contributors, advertisers, and sponsors, and do not represent those of JCEMS, the National Collegiate Emergency Medical Services Foundation, or any representatives, agents, or licensors. The appearance of advertisements does not represent a warranty, endorsement, or approval of the products or services advertised. JCEMS, the National Collegiate Emergency Medical Services Foundation, or any representatives, agents, or licensors make no warranties, representations, or other claims as to the accuracy or completeness of any articles or other content contained in JCEMS. JCEMS and NCEMSF disclaim responsibility for any injury to persons or property resulting from ideas, products, or other content referred to in articles or other content in JCEMS. EMS providers should always consult medical direction and local EMS protocols.

Instructions for Authors: Instructions for authors may be found in the JCEMS Guide for Authors at https://www.collegeems.com. Authors are required to abide by the latest guidelines available on-line at the time of submission. 


\title{
Inside the JCEMS Team
}

\section{Organizational Structure \& Opportunities for Involvement}

\author{
Nicholas M.G. Friedman, BA, EMT \& Brittany J. Dingler, MHS, PA
}

\begin{abstract}
JCEMS is proud to be managed by a team of all volunteers that includes recognized experts in prehospital emergency care as well as student-leaders. Here we offer an inside look at the organizational structure of JCEMS and share opportunities to join the team.
\end{abstract}

$\mathbf{T}$ he release of this issue (Volume 2, Issue 2) marks the point at which JCEMS has been in continuous publication for two years. Since the establishment of JCEMS, we have worked to carry out our mission of promoting leadership, clinical excellence, and original research. As the first peer-reviewed journal dedicated to collegiate- or campus-based EMS (CBEMS), we now fill a critical gap in the literature by publishing high quality CBEMS scholarship - from clinical reviews on drug-facilitated sexual assault to original research on university mass gathering events. As the official journal of the National Collegiate EMS Foundation (NCEMSF), we also support the next generation of EMS researchers by leading educational programs and events - from our Research Mentorship

Program to our annual Academic Poster Session.

As your Editors, the continued success and growth of JCEMS has been particularly humbling given the journal's rare status as an open access journal managed entirely by volunteers. From the student-leaders who manage day-to-day operations to the renowned experts on our Editorial Board, our team consists of dedicated volunteers who are spread across the United States. In this editorial we offer an inside look at our organizational structure and share opportunities for students and recent alumni to gain professional leadership experience in academic publishing.

\section{The Editors \& Editorial Board}

Consistent with standards for peer-reviewed medical journals, JCEMS is led by a team of Editors and Editorial Board members. The Editor-in-Chief (N.M.G.F.) and Executive Editor (B.J.D.) oversee management of day-to-day operations and maintain responsibility for the overall development and conduct of the journal. The Editorial Board assists the Editors in creating a vision for the journal, developing editorial policy, evaluating submitted manuscripts, and attracting talent and content. For example, an Editorial Board member might recommend a revision to our peer review policy or identify a promising candidate for a staff role.

Our Editorial Board is composed of emergency medicine phy-

Nicholas M.G. Friedman, BA, EMT is the Editor-in-Chief of JCEMS and a medical student at Stanford University.

Brittany J. Dingler, MHS, PA is the Executive Editor of JCEMS and a recent graduate of the Physician Assistant program at Drexel University.

\section{Key Points}

Established two years ago, JCEMS fills a critical gap in the scholarly literature and helps to develop the next generation of EMS researchers.

The team consists entirely of volunteers, including both recognized experts and student-leaders.

The Editorial Board, led by the Editors, creates a vision for the journal and develops editorial policy.

Student-leaders and recent alumni manage day-to-day operations and gain professional leadership experience in academic publishing.

sicians and other professionals with extensive records of service in the collegiate EMS community as well as expertise in prehospital emergency care research. Several members also bring their deep knowledge of academic publishing operations, developed through having served in editorial roles at other journals. We are exceedingly fortunate to have the depth of expertise that our Editorial Board members offer.

\section{Opportunities for Students \& Recent Alumni}

Unique amongst peer-reviewed medical journals, day-to-day publishing operations are run by part-time volunteers who are primarily students (graduate and undergraduate) or recent alumni. Volunteers serve in diverse roles, including: editorial management, business development, leadership of educational programs and special events, web development and design, layout and graphic design, news journalism, copy editing, marketing, and public relations. Given the critical roles they fill, students and recent alumni are carefully selected to join the team based on demonstrated leadership potential, relevant prior experience, and technical proficiency (when applicable for specific positions).

We are proud to rely on the contributions of students and recent alumni, and we are committed to supporting their professional de- 
velopment. Volunteers gain invaluable leadership and management experience with opportunities to assume progressively greater responsibilities over time. For volunteers focused on web development or graphic design, contributing to JCEMS also provides a route to hone technical skills and build a portfolio.

Importantly, all volunteers gain an insider's perspective of the academic publishing industry and have the opportunity to network with leaders in emergency medicine. Regardless of their specific roles, our volunteers meaningfully contribute to the growth of a rapidly-developing publication and find that the experience serves them well as they seek graduate education or advanced career opportunities.

\section{Continued Development of the JCEMS Team}

The continued growth and success of JCEMS has been made possible by the collective contributions of the JCEMS team members, each of whom graciously offers their passion, expertise, and time. To continue our record of success, we are always investigating strategies to optimize our ability to serve the CBEMS community. As JCEMS enters its third year of publication, we will be expanding our team with additional volunteers. We encourage inquiries from individuals who wish to learn more about opportunities for involvement, and we provide an up-to-date listing of open positions on our website. Our available positions and organizational structure may change over time, but our commitment to promoting CBEMS research and the professional development of our team members will always remain.

Author Affiliations: From The Journal of Collegiate Emergency Medical Services, National Collegiate Emergency Medical Services Foundation, West Sand Lake, NY, USA (B.J.D., N.M.G.F.).
Address for Correspondence: Nicholas M.G. Friedman, BA, EMT E-mail: JCEMS@CollegeEMS.com

Conflicts of Interest/Funding Sources: By the JCEMS Submission Declaration Form, all authors are required to disclose all potential conflicts of interest and funding sources. N.M.G.F. and B.J.D. serve in uncompensated editorial roles for JCEMS. All authors declared that they have no others conflicts of interest. All authors declared that they did not receive funding to conduct the research and/or writing associated with this work.

Authorship Criteria: By the JCEMS Submission Declaration Form, all authors are required to attest to meeting the four ICMJE.org authorship criteria: (1) Substantial contributions to the conception or design of the work; or the acquisition, analysis, or interpretation of data for the work; AND (2) Drafting the work or revising it critically for important intellectual content; AND (3) Final approval of the version to be published; AND (4) Agreement to be accountable for all aspects of the work in ensuring that questions related to the accuracy or integrity of any part of the work are appropriately investigated and resolved.

Submission History: Received December 31, 2019; accepted for publication December 31, 2019.

Published Online: December 31, 2019

Published in Print: December, 2019 (Volume 2: Issue 2)

Reviewer Information: In accordance with JCEMS editorial policy, Editorial manuscripts are reviewed by the JCEMS Editorial Board and published by the Editor-in-Chief or designee. JCEMS thanks the Editorial Board members who contributed to the review of this work.

Copyright: (C) 2019 Friedman \& Dingler. This is an open access article distributed under the terms of the Creative Commons Attribution 4.0 International (CC BY 4.0) License, which permits unrestricted use, distribution, and reproduction in any medium, provided the original author and source are credited. The full license is available at: https://creativecommons.org/licenses/by/4.0/

Electronic Link: https://doi.org/10.30542/JCEMS.2019.02.02.01

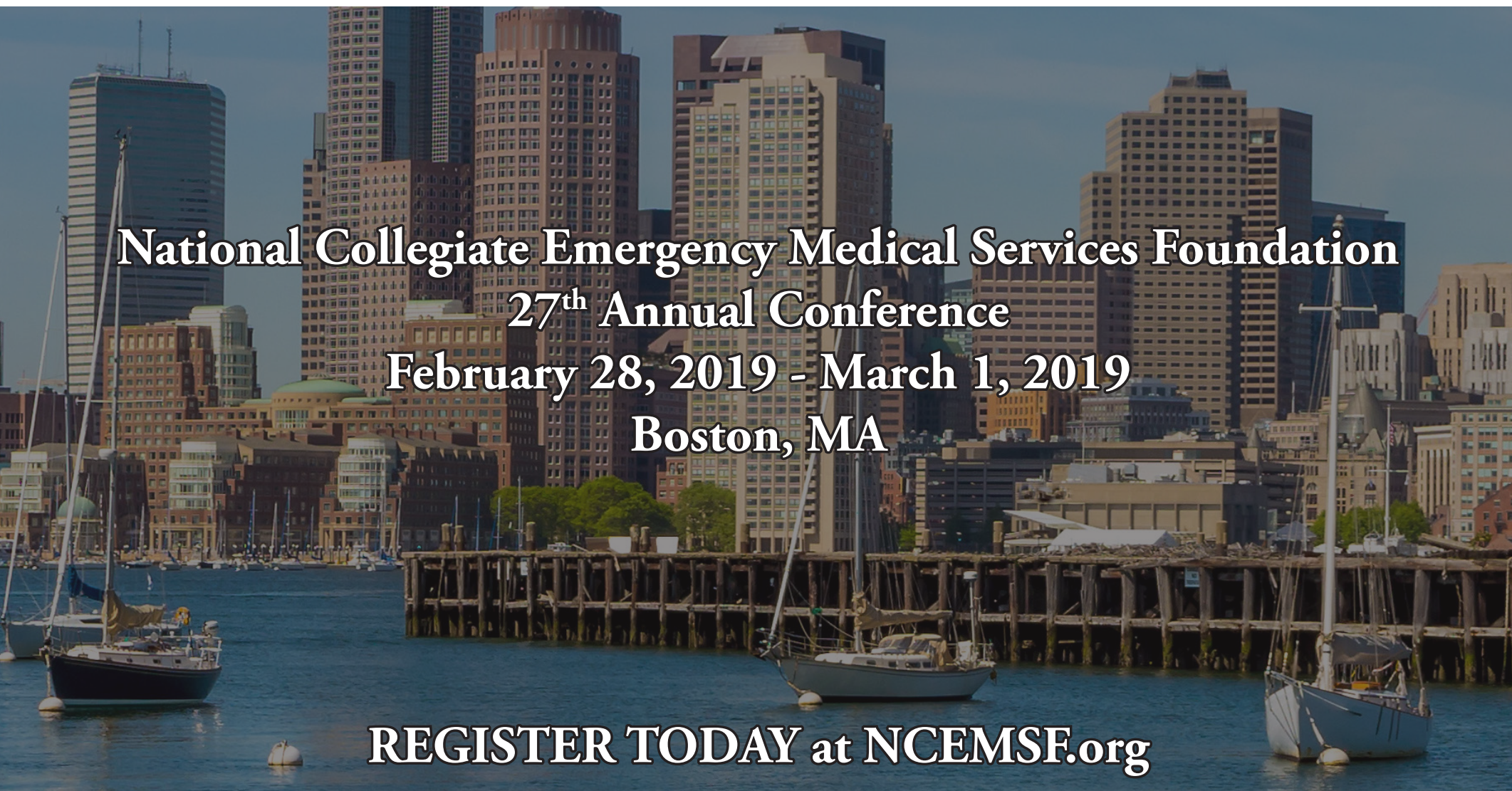




\title{
Interview with Paul Halliday: \\ 2019 Collegiate EMS Provider of the Year
}

\author{
JCEMS offers an exclusive interview with Case Western Reserve University (CWRU) EMS'S former \\ chief and innovative leader.
}

$\mathbf{P}$ aul Halliday was honored by the National Collegiate EMS Foundation (NCEMSF) as the 2019 Collegiate EMS Provider of the Year. At the $26^{\text {th }}$ Annual Conference of NCEMSF, in Pittsburgh, PA, he was cited for his outstanding leadership and service as the chief of Case Western Reserve University (CWRU) EMS. Chief Halliday was instrumental in developing several programs at CWRU EMS, including an innovative training system. $\mathrm{He}$ is currently enrolled in a paramedic program in the Cleveland area and works as an orthopedic trauma research coordinator for MetroHealth Medical Center. He plans to attend medical school next year. JCEMS had the opportunity to talk to Chief Halliday about leadership in EMS, his passion for prehospital care, and his future career plans.

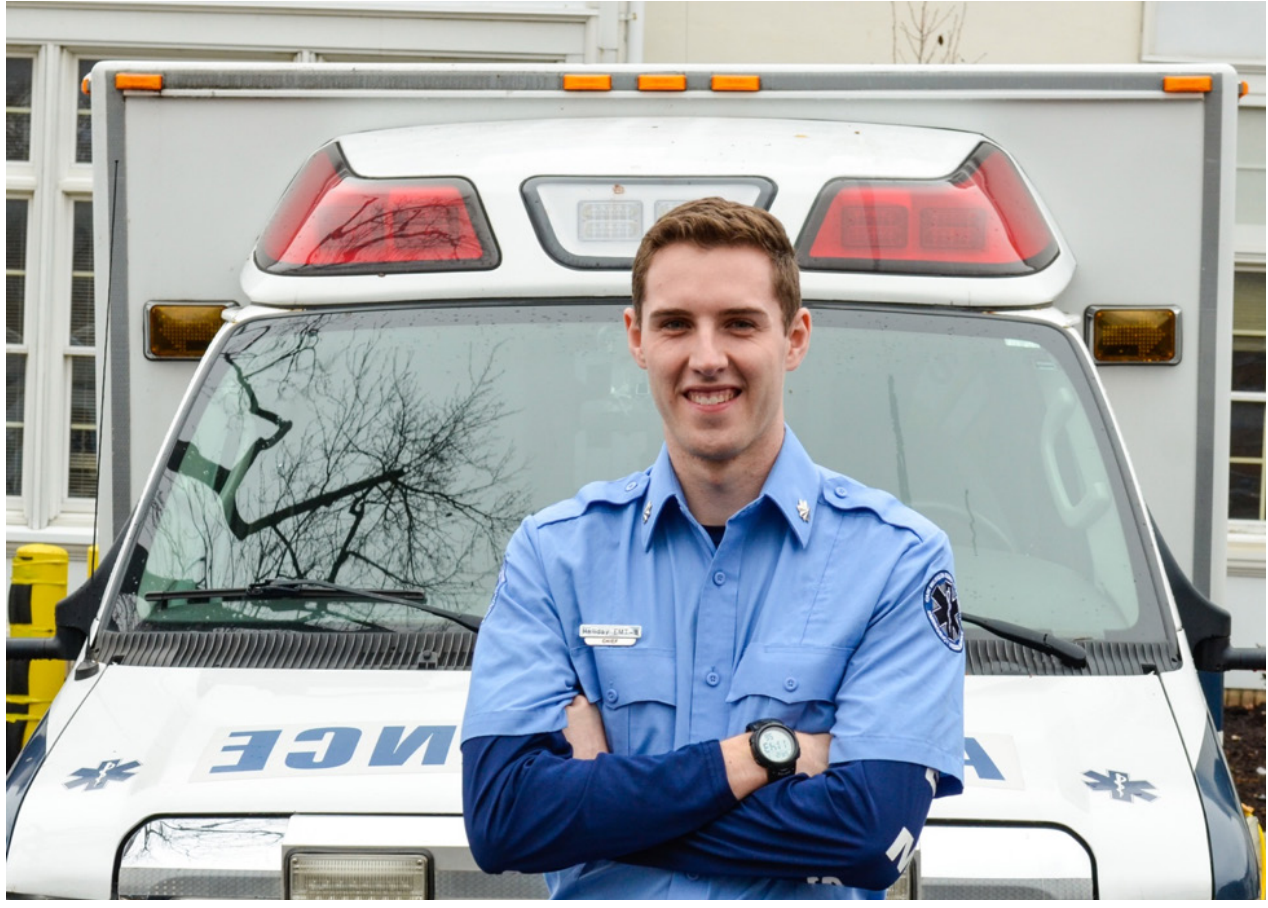

Paul Halliday, the NCEMSF 2019 Collegiate EMS Provider of the Year, stands at the ready in front of one of the Case Western Reserve University EMS ambulances (Cleveland, OH).

\section{Tell us about how you first got involved in EMS.}

I remember first seeing members of Case Western Reserve University (CWRU) EMS at an activities fair during my fist year of college. I had received my EMT certification before college and joining CWRU EMS seemed like an unparalleled opportunity to gain hands on experience with direct patient care - something many pre-meds struggle to find. A part of me also wanted to join solely to get to drive the ambulance! The next day I found their headquarters and that weekend I was already on a shift. My initial plans of running cross country that year didn't pan out, so I immersed myself in EMS and quickly rose through the ranks, learning as much as I could along the way. The amazing team at CWRU EMS kept me coming back every day to respond to emergencies at all hours of the night.

\section{As Collegiate EMS Provider of the Year, you were cited} for your exemplary leadership of numerous initiatives. What project are you most proud of?

While serving with CWRU EMS, I had the opportunity to help with a number of amazing programs including CPR education, Stop the Bleed, and first aid training. We made strides in growing our number of members, purchasing new vehicles, and buying new equipment. The most meaningful project I helped tackle, however, was developing a new training system. I believe EMS as a whole struggles to train new EMTs in ways that make them prepared for real scenarios. Crossing the bridge between textbook and real life is difficult and requires a comprehensive understanding of both clinical care and operations. We created a checklist-based training program that systematically trains members in all of our equipment and procedures, culminating with a number of realistic scenarios. While there will always be room to improve, this system has driven the organization forward and improved patient care and clinical skills.

\section{Leading an EMS organization as a student can present} intense challenges. What advice do you have for other leaders of collegiate EMS organizations?

Collegiate EMS is a unique subset of prehospital care and an incredible platform for training both leaders and providers. New collegiate 
EMS leaders should never be afraid to challenge "the way we've always done it," demand evidence-based medicine, or seek help from experts. It can be daunting to jump into the role of chief of a 50+ member organization and spark change. New leaders should keep in mind that we are all still learning and there is not just one right way to run an organization.

What are your future career goals? How has your collegiate EMS experience prepared you?

After completing paramedic school this year, I'll be heading to medical school. I hope that my experience in the field has given me a strong foundation in patient

"New collegiate EMS leaders should never be afraid to challenge 'the it,' demand evidencebased medicine, or seek help from experts." way we've always done assessment and hands on skills. CWRU EMS has taught me things no textbook could everything from learning you need a prescription for sterile water to how to stay calm while surrounded by five patients at a car accident. While both stressful and challenging, EMS has taught me to keep my cool in any situation and to adapt to whatever is thrown at me. If anything, working as an EMT has given me an appreciation for the importance of advanced prehospital care and the impact we can have on patient outcomes.
Author Affiliations: From Case Western Reserve University Emergency Medical Services, Cleveland, OH, USA (P.H.); The Journal of Collegiate Emergency Medical Services, National Collegiate EMS Foundation, West Sand Lake, NY, USA (N.MG.F.).

Address for Correspondence: [Interviewer] Nicholas M.G. Friedman, BA, EMT, The Journal of Collegiate Emergency Medical Services

E-mail: JCEMS@CollegeEMS.com

Conflicts of Interest/Funding Sources: By the JCEMS Submission Declaration Form, all authors are required to disclose all potential conflicts of interest and funding sources. N.MG.F. serves in an uncompensated editorial role for JCEMS. All authors declared that they have no others conflicts of interest. All authors declared that they did not receive funding to conduct the research and/or writing associated with this work.

Submission History: This interview was solicited by the JCEMS Editorial Board. Interview responses received December 22, 2019; accepted for publication December 23, 2019.

Published Online: December 31, 2019

Published in Print: December 31, 2019 (Volume 2: Issue 2)

Reviewer Information: In accordance with JCEMS editorial policy, interview responses are reviewed by the JCEMS Editorial Board. Interviews are published as submitted - save for copy-editing.

Copyright: (C) 2019 Halliday \& Friedman. This is an open access article distributed under the terms of the Creative Commons Attribution 4.0 International (CC BY 4.0) License, which permits unrestricted use, distribution, and reproduction in any medium, provided the original author and source are credited. The full license is available at: https://creativecommons.org/licenses/by/4.0/

Electronic Link: https://doi.org/10.30542/JCEMS.2019.02.02.02

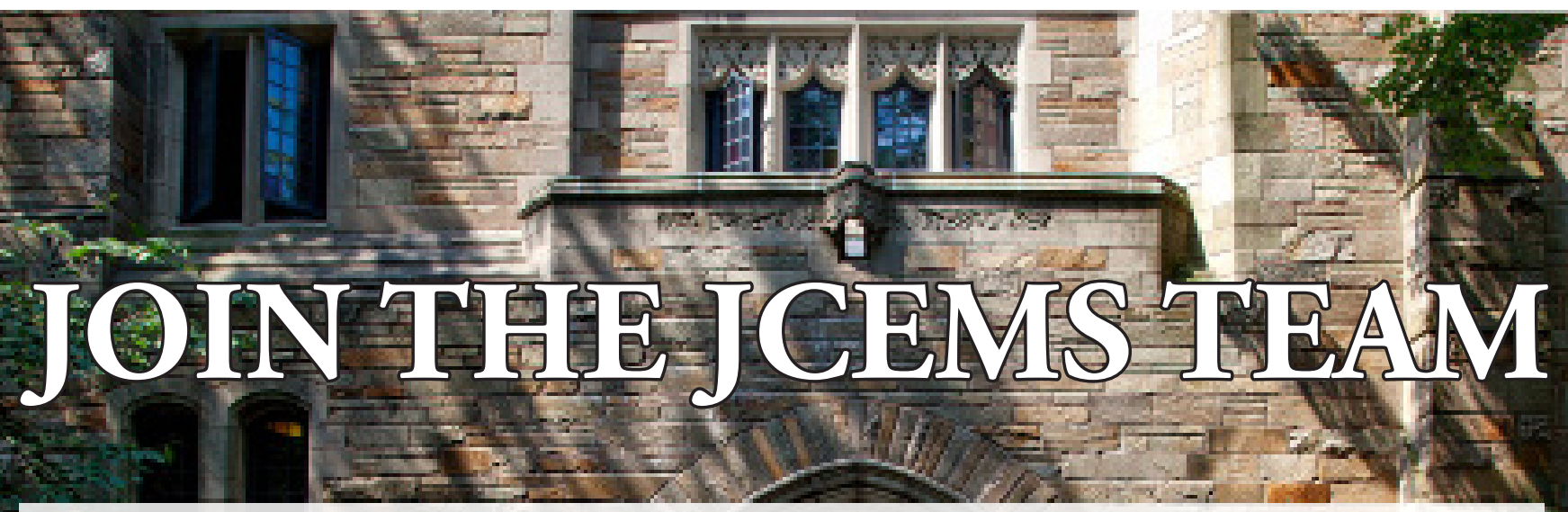

Now seeking motivated, passionate volunteers to join the JCEMS team. Available positions in the design, outreach, editing, and business management departments.

Excellent opportunity for current students and recent alumni to gain professional experience in an innovative start-up publication.

For current opportunities please email JCEMS@CollegeEMS.com or visit: https://www.collegeems.com/editorial-board/ 


\title{
Interview with Catherine Gibbs:
}

\section{Winner of the 2019 NCEMSF Vomacka Student Speaker Competition}

\author{
Catherine Gibbs shares her passion for community engagement and offers advice on how student \\ speakers can inspire an audience.
}

\section{You've been involved in EMS since an early age. Where did your EMS career begin?}

I started my EMS career at age twelve by enrolling in my local ambulance company's summer camp. I continued by joining the company's Explorer Post program, designed for adolescents interested in emergency service or medical careers. Through this program, I was able to run on the ambulance and obtain certifications such as Emergency Medical Responder and Emergency Medical Technician. I continued my EMS education and became one of the youngest Advanced Emergency Medical Technicians and EMS Instructors in Pennsylvania. I was also very engaged with the community and designed and coordinated many programs and fundraisers. I became a community course instructor and founded our company's Teddy Bear Hospital and Pre-Junior Medic Camp. Both programs are designed to engage children at an early age and promote the importance of safety and basic emergency preparedness and management.

\section{How did your early love of EMS help you serve in a leadership role in collegiate EMS?}

When I was applying to college, one of the top criteria was a collegiate EMS program with opportunities to teach. Temple University was fairly close to home, and I fell in love with their EMS program....oh, and the school too! After I began school, I joined Temple University's EMS program and eventually became the Director of Operations. Temple EMS offered a wonderful community and there were opportunities for the organization to grow. It was incredibly important to me to engage my new community in emergency preparedness, so I designed a tiered education program to engage school-aged children in emergency management and preparedness. The program, Basic Education of Aid and Rescue (BEAR), introduced more complex first-aid/CPR concepts as the students aged.

\section{Since graduating from Temple, have you remained active in EMS?}

I continue to work with Temple University, as an EMS Liaison, where I am working to develop an on- campus EMT program. I also continue to work with my original EMS company as an EMS educator and I work with Chester County Department of Emergency Services as an NREMT Exam Evaluator. Most recently, in addition to continuing my education at Thomas Jefferson, I've also begun to work as an EMT contractor for the Children's Hospital of Philadelphia Emergency Transport Team.

\section{Vomacka Student Speaker Competition Winner}

Catherine Gibbs was honored as the winner of The Richard W. Vomacka Student Speaker Competition at the $26^{\text {th }}$ Annual Conference of the National Collegiate EMS Foundation (NCEMFS) in Pittsburgh, PA. The Vomacka Competition named for an NCEMSF mentor who passed away in 2001 - was introduced in 2002 and offers students an opportunity to showcase their presentation skills with high quality educational seminars. Ms. Gibbs delivered a well-researched, inspiring presentation on the role of EMS in community and youth engagement. Ms. Gibbs graduated in 2019 with a B.S. in Neuroscience from Temple University (Philadelphia, PA), where she served as the Director of Operations for Temple EMS. She is currently continuing her education at Thomas Jefferson University (Philadelphia, PA) and aspires to pursue a career as a Prehospital Registered Nurse.

You won the Vomacka Student Speaker Competition for your presentation on community and youth engagement. Why was this an important topic to you?

Emergencies are unpredictable and can occur at any moment. Over 240 million 9-1-1 calls are made in the United States each year. The national average for an emergency response is approximately fifteen minutes. The overwhelming majority of community members are ill-prepared when an emergency arises. When it comes to saving lives, every second counts and a prepared bystander often make the difference between life and death. I think it is essential for all EMS organizations to engage with their communities, and especially youth, in order to teach lifesaving techniques and emergency prevention After attending college in an under-served part of Philadelphia, I gained an immense appreciation "Pick a topic you are passionate about. Your presentation should inspire your audience and they should feel your enthusiasm!" for the impact that even a small outreach program can have. My presentation for the Vomacka Student Speaker Competition was designed to help collegiate EMS organizations start community engagement programs and find ways to captivate their target audience. Based on my experience, I discussed 


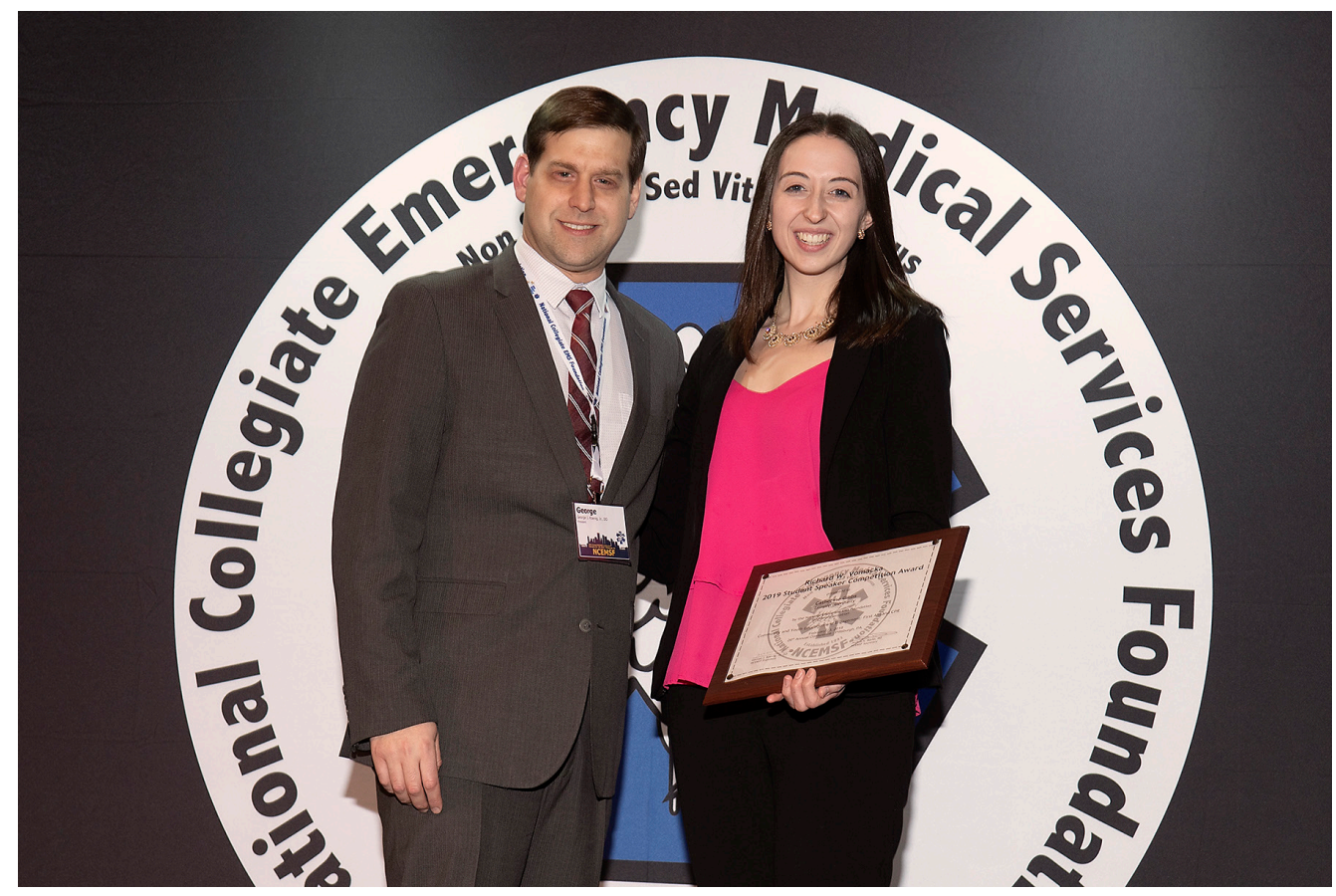

Catherine Gibbs wins the 2019 Vomacka Student Speaker Competition from Dr. George J. Koenig, President of the National Collegiate EMS Foundation (Pittsburgh, PA). grant funding. Most importantly, remember you will make a difference. Getting through to one person can have an immense impact.

\section{Any advice for students who are thinking about giving a presentation at the Vomacka Competition?}

Presenting at the 2019 Vomacka Competition last year was an immense honor and an absolute pleasure. I really enjoyed meeting the other speakers and hearing about their presentations. Their passion was evident and radiated through their conversation. My advice to anyone considering submitting a presentation is to make sure you pick a topic you are passionate about. Your presentation should inspire your audience and they should feel your enthusiasm!

how to target education based on individual learning styles, how to develop youth education programming, and how to promote longterm program success. As EMS providers, it's critical that we train bystanders to help prevent emergencies before they occur and to be prepared when they do.

\section{What advice do you have for collegiate EMS} organizations that want to get involved in community and youth engagement?

Every program can be successful. At the end of the day, it all comes down to the effort you put in. A great program will take time and effort. A lot of it. It is not always easy, but if you are passionate, giving up is never an option. And remember, keep revising to improve your program and help reach more people. Keep in mind, finding engagement tools does not have to be an expensive, extravagant endeavor. For example, my dad and I built a wooden ambulance out of supplies from a local hardware store to create an engaging toy for children. Do not be afraid

\section{"A great program} will take time and effort...If you are passionate, giving up is never an option... do not be afraid to ask for support!" to ask for support! Partnering with NCEMSF is a great way to connect with other collegiate organizations, but you can also connect with local departments. In regards to funding, sit down with your student government to request additional funding. Make sure you have a detailed proposal/outline prepared for the project. Do not be afraid to reach out to local companies and organizations; just make sure you have a professional letter drafted. Also, request to meet with your university's philanthropy department or apply for
Author Affiliations: From Temple University Emergency Medical Services, Temple University, Philadelphia, PA, USA (C.G.); The Journal of Collegiate Emergency Medical Services, National Collegiate EMS Foundation, West Sand Lake, NY, USA (N.MG.F.).

Address for Correspondence: [Interviewer] Nicholas M.G. Friedman, BA, EMT, The Journal of Collegiate Emergency Medical Services

E-mail: JCEMS@CollegeEMS.com

Conflicts of Interest/Funding Sources: By the JCEMS Submission Declaration Form, all authors are required to disclose all potential conflicts of interest and funding sources. N.MG.F. serves in an uncompensated editorial role for JCEMS. All authors declared that they have no others conflicts of interest. All authors declared that they did not receive funding to conduct the research and/or writing associated with this work.

Submission History: This interview was solicited by the JCEMS Editorial Board. Interview responses received December 23, 2019; accepted for publication December 24, 2019 .

Published Online: December 31, 2019

Published in Print: December 31, 2019 (Volume 2: Issue 2)

Reviewer Information: In accordance with JCEMS editorial policy, interview responses are reviewed by the JCEMS Editorial Board. Interviews are published as submitted - save for copy-editing.

Copyright: (C) 2019 Gibbs \& Friedman. This is an open access article distributed under the terms of the Creative Commons Attribution 4.0 International (CC BY 4.0) License, which permits unrestricted use, distribution, and reproduction in any medium, provided the original author and source are credited. The full license is available at: https://creativecommons.org/licenses/by/4.0/

Electronic Link: https://doi.org/10.30542/JCEMS.2019.02.02.03 


\title{
Development of an Excel-Based Software Solution to Collegiate EMS Shift Scheduling \& Placement
}

\author{
David Gordon, EMT; Ezra Brooks, EMT; Donavon Sandoval, EMT; Kaitlyn Boyle, EMT
}

POSTER PRESENTATION ABSTRACT | PROGRAM DEVELOPMENT \& EVALUATION CATEGORY

Introduction: For variably-scheduled volunteer EMS organizations, scheduling becomes a priority to sustain services to the community. EMS leadership must fill each shift by placing EMTs on shifts that best match their availability. With approximately 50 EMTs, 20 of whom lead calls, the University of Pennsylvania's Medical Emergency Response Team (MERT) covers 18 shifts per week, with 2-4 EMTs on each shift. Each week, MERT volunteers submit availability digitally. Prior to implementation of the current system, the scheduling coordinator would aggregate the schedule options submitted and assemble the schedule by hand. This system was inefficient and imperfect.

Program Development \& Implementation: While there are commercial solutions available, over Fall 2018, MERT developed an Excel-based solution customized to the agency's needs. The result is an Excel-spreadsheet-based algorithm that interprets the availability and optimizes the number of covered shifts based off availability responses. A binary variable was generated for each person and each potential shift. Constraints limited total shifts an EMT could take, defined a filled shift, and ensured that EMTs were only slated when they were available. This generated a linear model that the Excel OpenSolver add-on optimizes to preferentially fill shifts and match providers to empty shifts. The solved model is then interpreted by an array function with conditional formatting to output a formatted schedule. Additional options are available on the front-end to force the solver to provide for shifts with two crews, athletics events, and/or call reviews.

Program Evaluation: With implementation over 8 scheduling cycles, the model increased the proportion of fully covered shifts from $56 \%$ to $76 \%(P<0.03)$ and partial coverage from $85 \%$ to $97 \%$ when the schedule was released $(P<0.05)$. This model now allows maximal coverage when the schedule is first released and has dramatically reduced the time needed to manually schedule providers.

Discussion/Conclusions: A similar model could potentially be utilized by other collegiate EMS organizations, without cost, to optimize availability and maximize coverage.

Author Affiliations: Medical Emergency Response Team, University of Pennsylvania, Philadelphia, PA, USA

Address for Correspondence: David Gordon, EMT

E-mail: davgor@sas.upenn.edu

Conflicts of Interest/Funding Sources: By the JCEMS Submission Declaration Form, all authors are required to disclose all potential conflicts of interest and funding sources. The authors declared that they have no conflicts of interest. The authors declared that they received funding from University of Pennsylvania Division of Public Safety, University of Pennsylvania Division of the Vice Provost for University Life, and University of Pennsylvania Robert A. Fox Leadership Program for the program and/or research described in this abstract.

Ethical Compliance: The authors attest that the research associated with this abstract was conducted in accordance with the JCEMS Ethics Guidelines.

Submission History: Received January 6, 2019; accepted for presentation and publication February 11, 2019.

Poster Presentation: This abstract was presented as a poster at the Academic Poster Session of the $26^{\text {th }}$ Annual Conference of the National Collegiate Emergency Medical Services Foundation; February 23, 2019; Pittsburgh, PA, USA. The authors received the First Place Award for the Best Poster Presentation Competition.

Published Online: December 31, 2019
Published in Print: December 31, 2019 (Volume 2: Supplemental 1)

Reviewer Information: In accordance with JCEMS editorial policy, poster presentation abstracts undergo double-blind peer-review by at least two reviewers (JCEMS Editorial Board members and/or independent reviewers) prior to acceptance for presentation and publication. JCEMS thanks the anonymous reviewers who contributed to the review of this work.

Copyright: (C) 2019 Gordon, Brooks, Sandoval \& Boyle. This is an open access abstract distributed under the terms of the Creative Commons Attribution 4.0 International (CC BY 4.0) License, which permits unrestricted use, distribution, and reproduction in any medium, provided the original author and source are credited. The full license is available at: https://creativecommons.org/licenses/by/4.0/ Electronic Link: https://doi.org/10.30542/JCEMS.2019.02.S1.01 


\title{
Free of Charge: Analysis of an Urban Collegiate EMS Agency
}

\author{
Cade Herman, EMT-B; Allison Foster, EMT-B; Heather Scianneaux, NREMT-P; \\ Daniel Garrett, MD
}

POSTER PRESENTATION ABSTRACT | ORIGINAL RESEARCH CATEGORY

Introduction: Tulane Emergency Medical Services (Tulane EMS) is a student-staffed, basic life support (BLS), transporting EMS agency that provides 24/7, free-of-charge service to students, staff, and non-affiliates. We sought to identify the cost savings to Tulane students of activating Tulane EMS by estimating what patients would be charged if New Orleans EMS, the municipal advanced life support (ALS) service that would otherwise respond to on-campus calls, were activated instead. Tulane EMS provides free ground transport to local hospitals for students, staff, and non-affiliates.

Methods: Between August and December 2018, Tulane EMS responded to 325 activations. After excluding all cancellations and ALS transfers, a sample size of 267 was achieved. Per Tulane EMS protocol, a billing itemization form is completed after transfer of care to the emergency department, capturing baseline maintenance fees, applied interventions, equipment use, and transportation decision with a per-mile rate. Alcohol- or substance- related calls were identified on the form. To estimate what New Orleans EMS would bill for comparable service, itemized, line-item billing charges were obtained from a New Orleans EMS representative. To estimate what insured-patients would be charged, Tulane Student Health Insurance (T-SHIP) plan was utilized as the minimum coverage requirement for all students per university policy. Per T-SHIP, ambulance transports are out-of-network services which require meeting a $\$ 500$ deductible and $90 \%$ of remaining charges deemed to be "Usual and Customary" are covered; however, any alcohol- or substance-related transports are not covered. "Usual and Customary" charges were approximated as the total estimated amount that would be billed by New Orleans EMS. Using IBM ${ }^{\circ}$ SPSS $^{\bullet}$ Statistics Version 25, hypothetical charges for each call if New Orleans EMS were to provide the recorded services were calculated via summation code, adding all accrued charges over the course of each call.

Results: Over the study period, 33.1\% of Tulane EMS activations were alcohol- or substance related. On average, the estimated, hypothetical charge to a student if New Orleans EMS were to respond is $\$ 1328.43$ ( $\mathrm{SD}=\$ 370.78$, Range $=\$ 130.00-2219.00$ ). Per T-SHIP, this charge is the estimated cost to a student if the call was alcohol- or substance-related. If the chief complaint was otherwise, the estimated cost to a student who had not yet met their deductible would be, on average, $\$ 582.84$.

Discussion/Conclusions: Based on our findings, the presence of Tulane EMS may alleviate a significant financial burden from its patients. Several limitations of this study must be acknowledged. Data on patient demographics - including insurance coverage - were not collected, precluding accounting for insurance variability. In addition, billed charges may not always match what an insurance company deems to be "Usual and Customary." Lastly, New Orleans EMS has a larger scope of practice as an ALS agency and may apply different interventions. Notwithstanding these limitations, quantifying the financial impact of a collegiate EMS agency may be useful to demonstrate the organization's value to university administrators.

Author Affiliations: Tulane Emergency Medical Services, Tulane University, New Orleans, LA, USA

Address for Correspondence: Daniel Garrett, MD.

E-mail: dgarret@tulane.edu

Conflicts of Interest/Funding Sources: By the JCEMS Submission Declaration Form, all authors are required to disclose all potential conflicts of interest and funding sources. The author declared that they have no conflicts of interest. The author declared that they did not receive funding to conduct the program or research associated with this work.

Ethical Compliance: The authors attest that the research associated with this abstract was conducted in accordance with the JCEMS Ethics Guidelines.

Submission History: Received January 7, 2019; accepted for presentation and publication February 10, 2019.

Poster Presentation: This abstract was presented as a poster at the Academic Poster Session of the $26^{\text {th }}$ Annual Conference of the National Collegiate Emergency Medical
Services Foundation; February 23, 2019; Pittsburgh, PA, USA. The authors received the Second Place Award for the Best Poster Presentation Competition.

Published Online: December 31, 2019

Published in Print: December 31, 2019 (Volume 2: Supplemental 1)

Reviewer Information: In accordance with JCEMS editorial policy, poster presentation abstracts undergo double-blind peer-review by at least two reviewers (JCEMS Editorial Board members and/or independent reviewers) prior to acceptance for presentation and publication. JCEMS thanks the anonymous reviewers who contributed to the review of this work.

Copyright: (C) 2019 Herman, Foster, Scianneaux \& Garrett. This is an open access abstract distributed under the terms of the Creative Commons Attribution 4.0 International (CC BY 4.0) License, which permits unrestricted use, distribution, and reproduction in any medium, provided the original author and source are credited. The full license is available at: https://creativecommons.org/licenses/by/4.0/ Electronic Link: https://doi.org/10.30542/JCEMS.2019.02.S1.02 


\title{
SIREN: Schema In-Fill Rendered from Entry Notifications
}

\author{
Elizabeth DeTienne, EMT; Milka Piszczek
}

POSTER PRESENTATION ABSTRACT | PROGRAM DEVELOPMENT \& EVALUATION CATEGORY

Introduction: Entry notes are radio communications between en-route first responders and a destination hospital to prepare hospital personnel for ambulance arrival. Ambulance services currently have no way to efficiently search entry note data or use it for training. The destination hospital may quickly lose the transmitted information, as it is reliant on a single listener. SIREN is an end-to-end tool that takes voice files of hospital entry notes as input and converts them into informative database tuples.

Program Development \& Implementation: SIREN takes in audio recordings of entry notes. The audio files are filtered using PySoX, by adding a low-pass filter, normalization, and a gain, which help remove background noise. The filtered audio is then transcribed using Google Cloud. From there, we extract the mentioned drugs using a dictionary of drug names, the chief complaints using a dictionary with associated symptoms and terminology, and the sex directly from the transcript. Parsing age is a non-trivial task since there's a large variance in the input (i.e. two-one vs. twenty one) and sometimes even repetition with the intent to clarify. We use Stanford's Natural Language Processing suite to extract cardinal numbers that are close to age-related words, and then we use a series of rules to extract the correct spoken age. We then put these into a database, which is visualized in a user-friendly table for skimming or searching.

Program Evaluation: We found that the audio transcript was sometimes cut short when a siren began in the background. Our filtering produced an incredible 50\% increase in the median number of words transcribed. We manually verified the accuracy of a series of SIREN transcripts. Transcription accuracy was generally excellent, and we found Google's transcription errors to be our biggest bottleneck. A typical transcription has only minor mistakes that do not affect meaning. Leadership in MIT EMS noted that this would help elevate entry note skill, by giving EMTs a database they could easily and enjoyable peruse, or use to look back at their own performance.

Discussion/Conclusions: We see two potential applications for SIREN: (1) SIREN can train current EMS providers to give professional and useful entry notes, either on their peers' or their own entry notes; and (2) SIREN can be adapted to process data in real-time, creating a reference that hospital staff can use to improve continuity of care. We hope that this can be a useful tool for collegiate EMS in the future. Plans for future improvement include making SIREN real-time or using machine learning to train the audio-to-text with EMS-specific language.

Author Affiliations: Massachusetts Institute of Technology (MIT) Emergency Medical Services, MIT, Cambridge, MA, USA

Address for Correspondence: Elizabeth DeTienne, EMT.

E-mail: elizdet@mit.edu

Conflicts of Interest/Funding Sources: By the JCEMS Submission Declaration Form, all authors are required to disclose all potential conflicts of interest and funding sources. The authors declared that they have no conflicts of interest. The authors declared that they did not receive funding to conduct the program or research associated with this work.

Ethical Compliance: The authors attest that the research associated with this abstract was conducted in accordance with the JCEMS Ethics Guidelines.

Submission History: Received December 12, 2018; accepted for presentation and publication February 17, 2019.

Poster Presentation: This abstract was presented as a poster at the Academic Poster Session of the $26^{\text {th }}$ Annual Conference of the National Collegiate Emergency Medical Services Foundation; February 23, 2019; Pittsburgh, PA, USA. The authors received the Third Place Award for the Best Poster Presentation Competition.

Published Online: December 31, 2019

Published in Print: December 31, 2019 (Volume 2: Supplemental 1)

Reviewer Information: In accordance with JCEMS editorial policy, poster presen- tation abstracts undergo double-blind peer-review by at least two reviewers (JCEMS Editorial Board members and/or independent reviewers) prior to acceptance for presentation and publication. JCEMS thanks the anonymous reviewers who contributed to the review of this work.

Copyright: $\odot 2019$ DeTienne \& Piszczek. This is an open access abstract distributed under the terms of the Creative Commons Attribution 4.0 International (CC BY 4.0) License, which permits unrestricted use, distribution, and reproduction in any medium, provided the original author and source are credited. The full license is available at: https://creativecommons.org/licenses/by/4.0/

Electronic Link: https://doi.org/10.30542/JCEMS.2019.02.S1.03 


\title{
No IV, No Problem: A Cross Sectional Analysis of Antiemetic Therapies in Statewide EMS Protocols
}

\author{
Carlin C. Chuck, NREMT; Thomas J. Martin, BA, NRP; Isabelle H. Moseley, NREMT; \\ Megan L. Ranney, MD, MPH, FACEP; Nicholas Asselin, DO, MS
}

POSTER PRESENTATION ABSTRACT | ORIGINAL RESEARCH CATEGORY

Introduction: Nausea is a common reason for ED presentation and may represent up to $10 \%$ of chief complaints in the prehospital setting. In the collegiate setting, EMS providers often encounter nausea secondary to consumption of alcohol or other drugs. The prehospital management of nausea varies with local protocol and scope of practice. We hypothesized that CBEMS agencies operating at the BLS level may have limited options for antiemetic therapy.

Methods: A cross-sectional analysis of publicly available statewide EMS protocols published within the last ten years was completed in October 2018. The presence of a BLS nausea protocol was defined as the primary outcome of interest; secondary outcomes including the availability of oral (ODT) ondansetron or alternative antiemetic agents were defined a priori. Data were collected independently by two trained reviewers using standardized abstraction forms; inter-rater reliability was assessed using Cohen's kappa ( $\kappa)$ and discrepancies were resolved by the senior author. Descriptive statistics were generated using R v3.3.2. This study was exempt from IRB review.

Results: 30 model or mandatory statewide EMS protocols were identified using aggregate databases and internet searches; data were abstracted with $\kappa=1$. Of the thirty states, $13.3 \%$ (4/30) have adopted BLS nausea protocols: two allow ODT ondansetron, one allows isopropyl alcohol aromatherapy, and one allows P6 acupressure. No protocols were identified that include intramuscular antiemetics for BLS providers. In addition, 70\% (21/30) of state protocols include ODT ondansetron, but 90.4\% (19/21) of these restrict administration to ALS providers. 46.7\% (14/30) of states include alternative antiemetics for ALS providers such as promethazine and metoclopramide, often as second-line therapies.

Discussion/Conclusions: CBEMS agencies operating at the BLS level are unable to administer ondansetron intravenously or intramuscularly. Though ODT ondansetron and isopropyl alcohol aromatherapy are safe and effective for treatment of nausea in the ED, prehospital adoption remains low. Further research should assess the feasibility, safety, and efficacy of introducing these therapies at the BLS level.

Author Affiliations: From Brown University Emergency Medical Services, Brown University, Providence, RI, USA (C.C.C, T.J.M, I.H.M, N.A.); The Warren Alpert Medical School, Brown University, Providence, RI, USA (T.J.M, M.L.R, N.A.); Program in Emergency Digital Health Innovation, Department of Emergency Medicine, The Warren Alpert Medical School, Brown University, Providence, RI, USA (M.L.R); Division of EMS, Department of Emergency Medicine, The Warren Alpert Medical School, Brown University, Providence, RI, USA (N.A.).

Address for Correspondence: Thomas J. Martin, BA, NRP.

Email: thomas_martin@brown.edu

Conflicts of Interest/Funding Sources: By the JCEMS Submission Declaration Form, all authors are required to disclose all potential conflicts of interest and funding sources. The authors declared that they have no conflicts of interest. The authors declared that they did not receive funding to conduct the program or research associated with this work.

Ethical Compliance: The authors attest that the research associated with this abstract was conducted in accordance with the JCEMS Ethics Guidelines.

Submission History: Received November 30, 2018; accepted for presentation and publication January 22, 2019.

Poster Presentation: This abstract was presented as a poster at the Academic Poster Session of the $26^{\text {th }}$ Annual Conference of the National Collegiate Emergency Medical
Services Foundation; February 23, 2019; Pittsburgh, PA, USA.

Published Online: December 31, 2019

Published in Print: December 31, 2019 (Volume 2: Supplemental 1)

Reviewer Information: In accordance with JCEMS editorial policy, poster presentation abstracts undergo double-blind peer-review by at least two reviewers (JCEMS Editorial Board members and/or independent reviewers) prior to acceptance for presentation and publication. JCEMS thanks the anonymous reviewers who contributed to the review of this work.

Copyright: (C) 2019 Chuck, Martin, Moseley, Ranney \& Asselin. This is an open access abstract distributed under the terms of the Creative Commons Attribution 4.0 International (CC BY 4.0) License, which permits unrestricted use, distribution, and reproduction in any medium, provided the original author and source are credited. The full license is available at: https://creativecommons.org/licenses/by/4.0/ Electronic Link: https://doi.org/10.30542/JCEMS.2019.02.S1.04 


\title{
Do EMT students enrolled through a college course perform better in terms of course completion and exam success?
}

\author{
Daniel Armstrong, DPT, MS \& Kevin Rummel, MS, RN, NRP
}

POSTER PRESENTATION ABSTRACT | ORIGINAL RESEARCH CATEGORY

Introduction: This project was designed to determine the difference in student course completion rates and certifying exam scores in college vs. non-college formats. College students often have access to academic and student support resources which can include tutoring, libraries, computer labs, writing and math centers, counseling services, and services for students with disabilities. We hypothesized that EMT students enrolled through a college format are more likely to complete an EMT course and score higher on the state certifying exam.

Methods: A retrospective review of an EMT program that offered an EMT class both through a college as a credited course and as a non-credited course through continuing education was conducted. Both sets of students experienced the same course. Regardless of enrollment type the students were mixed into the same class. Five years of student data from 2013 to 2018 was examined retrospectively. A total of 350 student records were examined. Course completion rates were analyzed with descriptive statistics and compared with a z-test for two population proportions. State certifying exam scores were analyzed with descriptive statistics and compared with a two tailed independent sample t-test. The level of statistical significance $(\alpha)$ was set at 0.05 .

Results: Over the 5 years analyzed, 181 (51.71\%) students were enrolled through the college and 169 (48.29\%) students were enrolled in the continuing education department. There was no significant difference in course completion rates between the proportion of students enrolled through the college $(54.67 \%)$ and through continuing education $(62.13 \%)(P=0.16)$. The mean score on the state certifying exam was significantly lower for students enrolled through the college $(77.42, \mathrm{SD}=6.91)$ than for students enrolled through continuing education $(79.95, \mathrm{SD}=8.32)(P=0.02)$.

Discussion/Conclusions: Contrary to our hypothesis, students enrolled through the college scored lower on the state certifying exam and there was no statistical difference in course completion between the groups. Further research is needed to determine if course completion rates and exam scores differ between college-enrolled and non-college-enrolled students at other EMT programs. Further research is also needed to determine which resources improve student success so collegiate program directors can encourage students to utilize them.

Author Affiliations: Health, Physical Education, and Dance Department, Queensborough Community College, Queens, NY, USA.

Address for Correspondence: Daniel Armstrong, DPT, MS.

Email: darmstrong@qcc.cuny.edu

Conflicts of Interest/Funding Sources: By the JCEMS Submission Declaration

Form, all authors are required to disclose all potential conflicts of interest and funding sources. The authors declared that they have no conflicts of interest. The authors declared that they did not receive funding to conduct the program or research associated with this work.

Ethical Compliance: The authors attest that the research associated with this abstract was conducted in accordance with the JCEMS Ethics Guidelines.

Submission History: Received November 30, 2018; accepted for presentation and publication January 31, 2019.

Poster Presentation: This abstract was presented as a poster at the Academic Poster Session of the $26^{\text {th }}$ Annual Conference of the National Collegiate Emergency Medical Services Foundation; February 23, 2019; Pittsburgh, PA, USA.

Published Online: December 31, 2019

Published in Print: December 31, 2019 (Volume 2: Supplemental 1)

Reviewer Information: In accordance with JCEMS editorial policy, poster presentation abstracts undergo double-blind peer-review by at least two reviewers (JCEMS
Editorial Board members and/or independent reviewers) prior to acceptance fo r presentation and publication. JCEMS thanks the anonymous reviewers who contributed to the review of this work.

Copyright: ( 12019 Armstrong \& Rummel. This is an open access abstract distributed under the terms of the Creative Commons Attribution 4.0 International (CC BY 4.0) License, which permits unrestricted use, distribution, and reproduction in any medium, provided the original author and source are credited. The full license is available at: https://creativecommons.org/licenses/by/4.0/

Electronic Link: https://doi.org/10.30542/JCEMS.2019.02.S1.05 


\title{
Reducing Barriers to Bystander Intervention: Piloting a Wintersession Program
}

\author{
Truelian Lee, EMT-B
}

POSTER PRESENTATION ABSTRACT | PROGRAM DEVELOPMENT \& EVALUATION CATEGORY

Introduction: Occurring during the last week of winter recess, Harvard University's Wintersession offers enrichment programming. Similar programs exist in various other colleges, including Brown University, Wellesley College, and Ithaca College. Harvard Crimson EMS saw Wintersession as an opportunity to reach students who are too busy during the school year to learn bystander intervention skills. Consequently, the organization piloted a series of Wintersession workshops for the first time this year.

Program Development \& Implementation: Over the period of a month, Crimson EMS planned workshops that occurred from 10 AM-3 PM on January 22-25, 2019. Students earned national certifications in AHA CPR Heartsaver/First Aid and Stop the Bleed. Crimson EMS instructors used a curriculum based on prior outreach efforts. Lectures were conducted with PowerPoint presentations and skills practice with manikins or with partners and instructors. The total budget for the program was $\$ 450$. Registration was capped at 15 students based on the number of available instructors. Crimson EMS primarily publicized the program through social media.

Program Evaluation: 14 students attended. Crimson EMS solicited free response feedback as well as Likert scale ratings with 1 representing "poor" and 5 "excellent" to evaluate the program. At least $40 \%$ of participants had no prior experience to the skills taught. On average, participants rated the overall program a $4.2(\mathrm{SD}=0.8)$, with the ratings for specific workshops ranging from 3.9 ( $\mathrm{SD}=1.4)$ for common collegiate EMS cases to $4.8(\mathrm{SD}=0.3)$ for cardiopulmonary resuscitation. Participants gave the lectures a $3.9(\mathrm{SD}=1.1)$ and the skills practice sections a $5.0(\mathrm{SD}=0.0)$.

Discussion/Conclusions: Based on participant responses, we plan to include additional skills training in future workshops and publicize the program through more platforms and for a longer period of time. This Wintersession represents a new avenue for Crimson EMS to promote collegiate EMS and educate community members about important, life-saving skills to reduce the barrier to bystander intervention.

\footnotetext{
Author Affiliations: Crimson EMS, Harvard University, Cambridge, MA, USA Address for Correspondence: Truelian Lee, EMT-B.

Email: truelianlee@college@harvard.edu

Conflicts of Interest/Funding Sources: By the JCEMS Submission Declaration Form, all authors are required to disclose all potential conflicts of interest and funding sources. The authors declared that they have no conflicts of interest. The authors declared that they received funding from Harvard University Undergraduate Council. Ethical Compliance: The authors attest that the research associated with this abstract was conducted in accordance with the JCEMS Ethics Guidelines.

Submission History: Received January 29, 2019; accepted for presentation and publication February 8, 2019.

Poster Presentation: This abstract was presented as a poster at the Academic Poster Session of the $26^{\text {th }}$ Annual Conference of the National Collegiate Emergency Medical Services Foundation; February 23, 2019; Pittsburgh, PA, USA.

Published Online: December 31, 2019

Published in Print: December 31, 2019 (Volume 2: Supplemental 1)

Reviewer Information: In accordance with JCEMS editorial policy, poster presentation abstracts undergo double-blind peer-review by at least two reviewers (JCEMS Editorial Board members and/or independent reviewers) prior to acceptance for presentation and publication. JCEMS thanks the anonymous reviewers who contributed to the review of this work.
}

Copyright: $\odot 2019$ Lee. This is an open access abstract distributed under the terms of the Creative Commons Attribution 4.0 International (CC BY 4.0) License, which permits unrestricted use, distribution, and reproduction in any medium, provided the original author and source are credited. The full license is available at: https://creativecommons.org/licenses/by/4.0/

Electronic Link: https://doi.org/10.30542/JCEMS.2019.02.S1.06 


\title{
Integrating Continuing Education Credits to a Collegiate EMS Training Model
}

\author{
David Gordon, EMT; Maya Ganeshan, EMT; Eugene Janda, MES, CFPS, NFPA; Laura Farrington
}

POSTER PRESENTATION ABSTRACT | PROGRAM DEVELOPMENT \& EVALUATION CATEGORY

Introduction: Ongoing continuing medical education (CME) is an important consideration for collegiate EMS programs because an EMT may need to recertify (every 2 or 3 years) during their collegiate EMS tenure. At the same time, the frequent turnover of training leadership roles may prevent students from being state-certified EMS instructors. In Pennsylvania, providers need 24 pre-approved online or in-person CME hours to recertify, which providers may complete independently from agency-specific training.

Program Development \& Implementation: The University of Pennsylvania Medical Emergency Response Team (MERT) develops 1015 student-led training drills per academic semester, which each EMT attends. Those unable to attend are required to complete Pennsylvania-CME online to make up the missed training. MERT sought a solution that positively encouraged EMTs to attend agency-specific training while providing CME in a matter logistically feasible for collegiate EMS agencies. For training drills, MERT recruited paramedics from Public Safety and physicians from the University Health System to assist in planning and mentoring students on a range of EMS topics. MERT leveraged a close partnership with the Division of Public Safety's Fire and Emergency Services (FES) and their regional-EMS teaching certificate agency to actuate the CME training sessions. Following drills, the training coordinator works closely with FES to submit CME credit approval paperwork to regional-EMS.

Program Evaluation: In October 2018, MERT piloted the CMEs and organized a symposium with 6 guest speakers attended by 50 providers from 4 collegiate EMS agencies. With plans to integrate at least 8 CME credits into MERT's training semester curriculum, providers who attended each required training will complete all credits necessary to recertify. So far, most providers elected to enroll in the CMEs.

Discussion/Conclusions: Other collegiate EMS agencies may potentially be able to utilize teaching certificates from their hospitals and public safety to adapt their training programs for members to ensure compliance with the CME licensing requirements.

Author Affiliations: From Medical Emergency Response Team, University of Pennsylvania, Philadelphia, PA, USA (D.G., M.Y.); Fire and Emergency Services, Division of Public Safety, University of Pennsylvania, Philadelphia, PA, USA (E.J., L.F.).

Address for Correspondence: David Gordon, EMT.

Email: davgor@sas.upenn.edu

Conflicts of Interest/Funding Sources: By the JCEMS Submission Declaration Form, all authors are required to disclose all potential conflicts of interest and funding sources. The authors declared that they received funding from the University of Pennsylvania Division of Public Safety, University of Pennsylvania Division of the Vice Provost for University Life, and University of Pennsylvania Robert A. Fox Leadership Program for the program and/or research described in this abstract.

Ethical Compliance: The authors attest that the research associated with this abstract was conducted in accordance with the JCEMS Ethics Guidelines.

Submission History: Received January 6, 2019; accepted for presentation and publication February 11, 2019.

Poster Presentation: This abstract was presented as a poster at the Academic Poster Session of the $26^{\text {th }}$ Annual Conference of the National Collegiate Emergency Medical Services Foundation; February 23, 2019; Pittsburgh, PA, USA.

Published Online: December 31, 2019

Published in Print: December 31, 2019 (Volume 2: Supplemental 1)
Reviewer Information: In accordance with JCEMS editorial policy, poster presentation abstracts undergo double-blind peer-review by at least two reviewers (JCEMS Editorial Board members and/or independent reviewers) prior to acceptance for presentation and publication. JCEMS thanks the anonymous reviewers who contributed to the review of this work.

Copyright: (๑) 2019 Gordon, Ganeshan, Janda \& Farrington. This is an open access abstract distributed under the terms of the Creative Commons Attribution $4.0 \mathrm{In}$ ternational (CC BY 4.0) License, which permits unrestricted use, distribution, and reproduction in any medium, provided the original author and source are credited. The full license is available at: https://creativecommons.org/licenses/by/4.0/ Electronic Link: https://doi.org/10.30542/JCEMS.2019.02.S1.07 


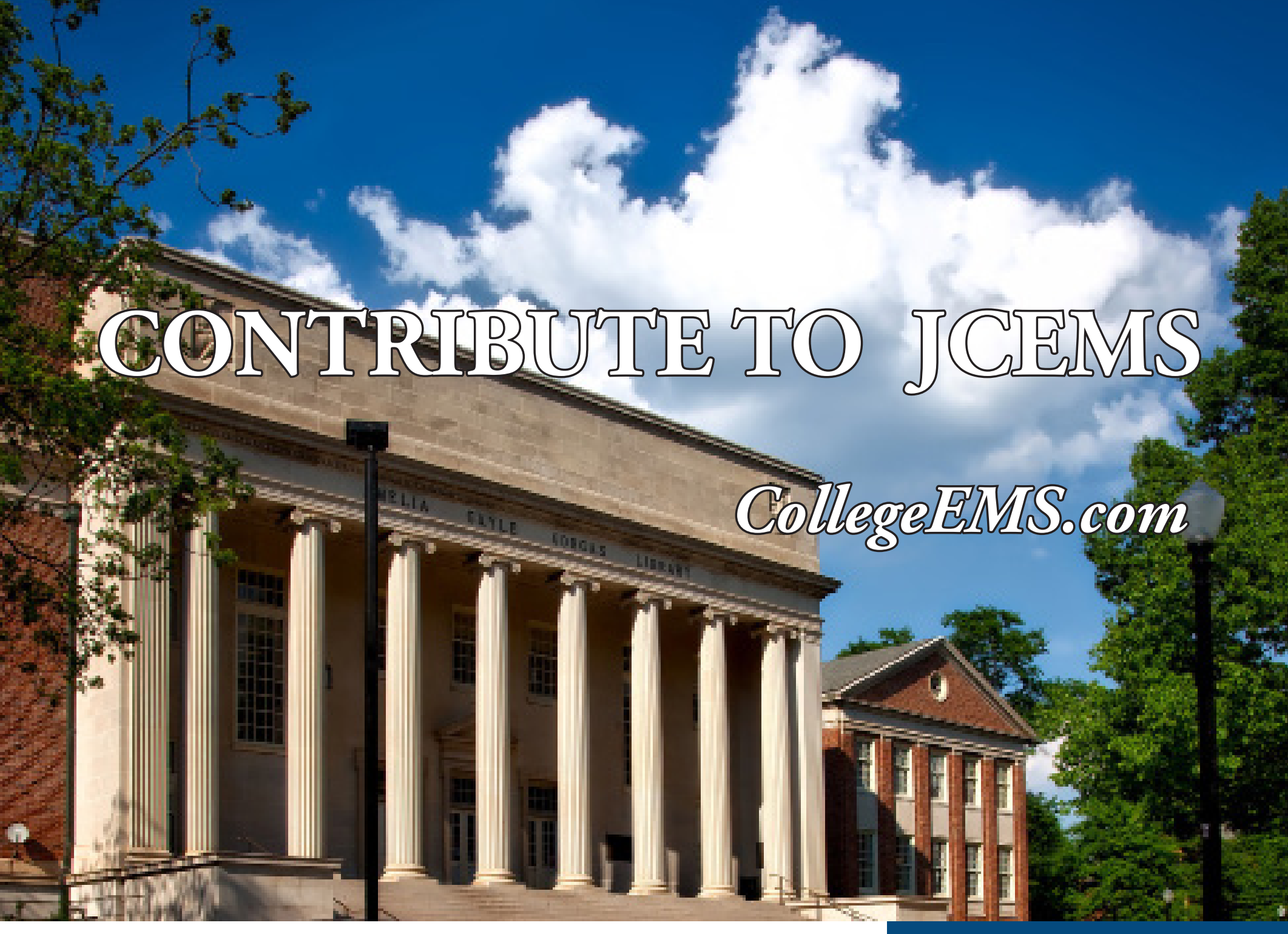

Submit to JCEMS: the official scholarly, peer-reviewed journal of the National Collegiate EMS Foundation.
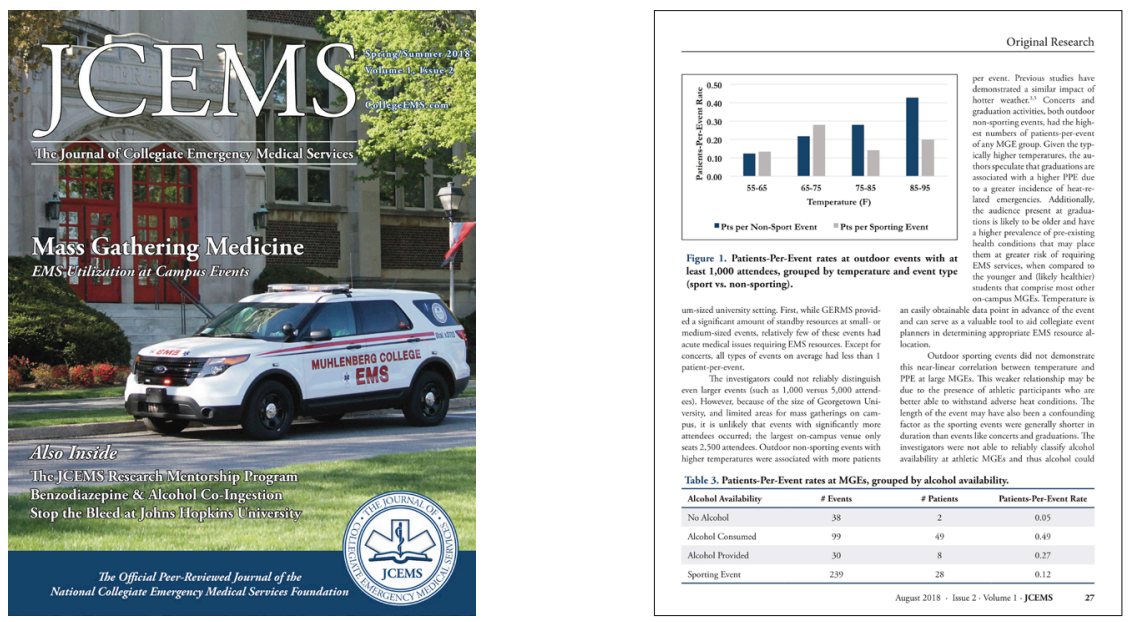

Original research is prioritized. Case reports, reviews, and articles featuring perspectives and commentary are also invited.

\section{Research Mentorship}

We are committed to mentoring student researchers and authors. If you might be interested in conducting a study or submitting a manuscript for publication, consider applying to the JCEMS Research Mentorship Program. Additional details are available at CollegeEMS.com.

\section{Submission Guidelines}

View submission guide at: CollegeEMS.com

Send inquiries to: JCEMS@CollegeEMS.com 


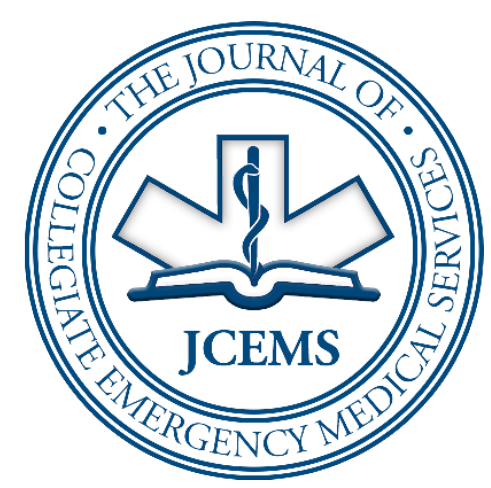

The Journal of

COLLEGIATE EMERGENCY MEDICAL SERVICES

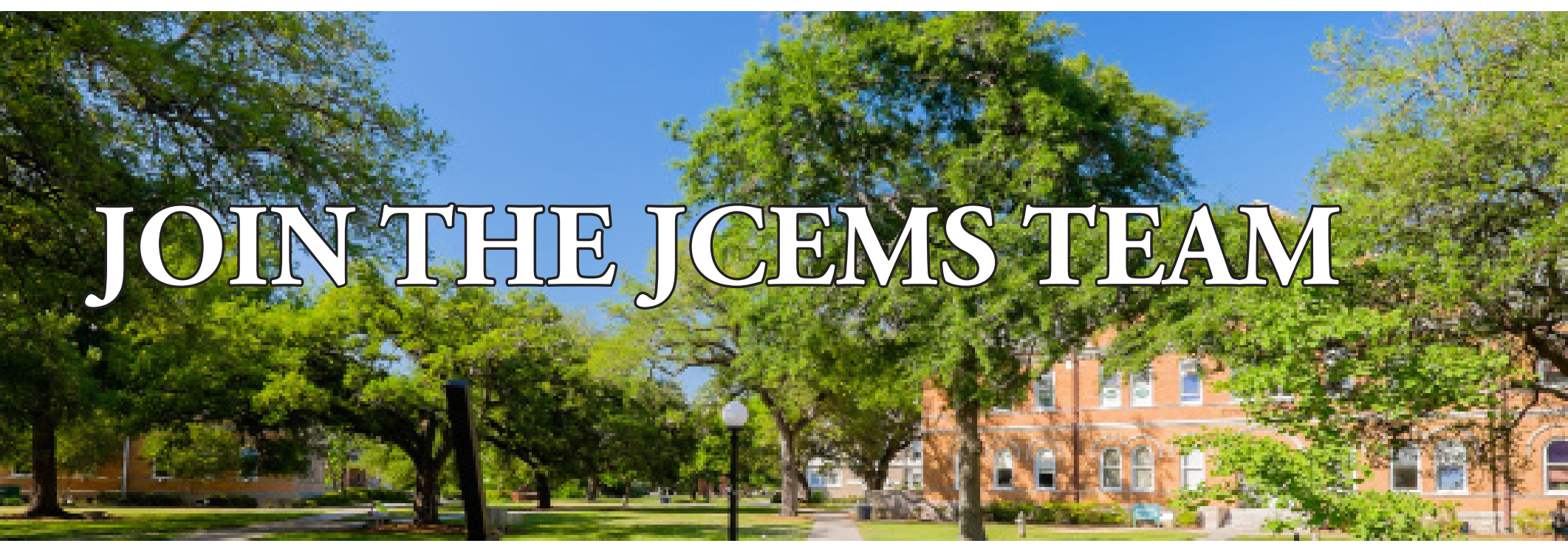

Now seeking motivated, passionate volunteers to join the JCEMS team. Available positions in the design, editing, outreach, and business management departments:

- Journalism \& Publishing

- $\quad$ Print \& Web Design

- $\quad$ Peer Review \& Copy-Editing

- Public Relations \& Social Media

- $\quad$ Advertising \& Marketing

Excellent opportunity for current students and recent alumni to gain professional leadership experience in an innovative, scholarly publication.

For open positions, please email JCEMS@collegeEMS.com or visit: https://www.collegeems.com/editorial-board/

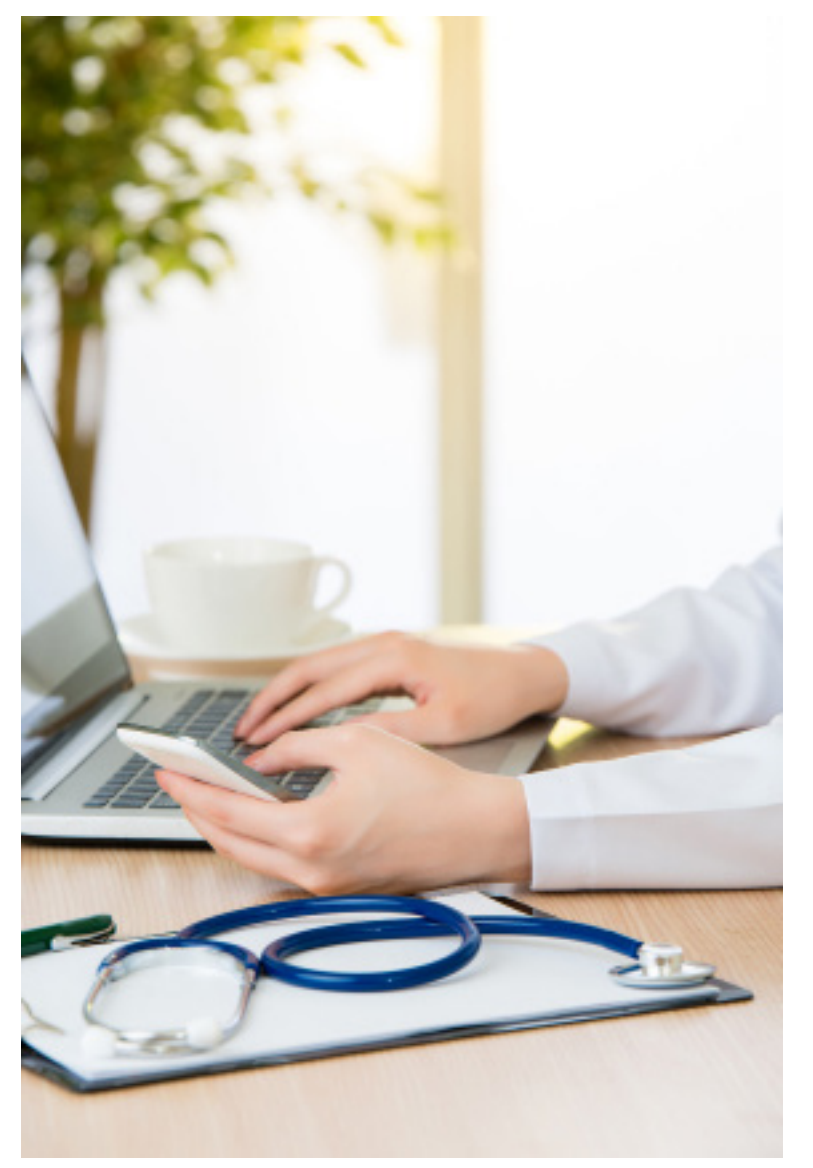




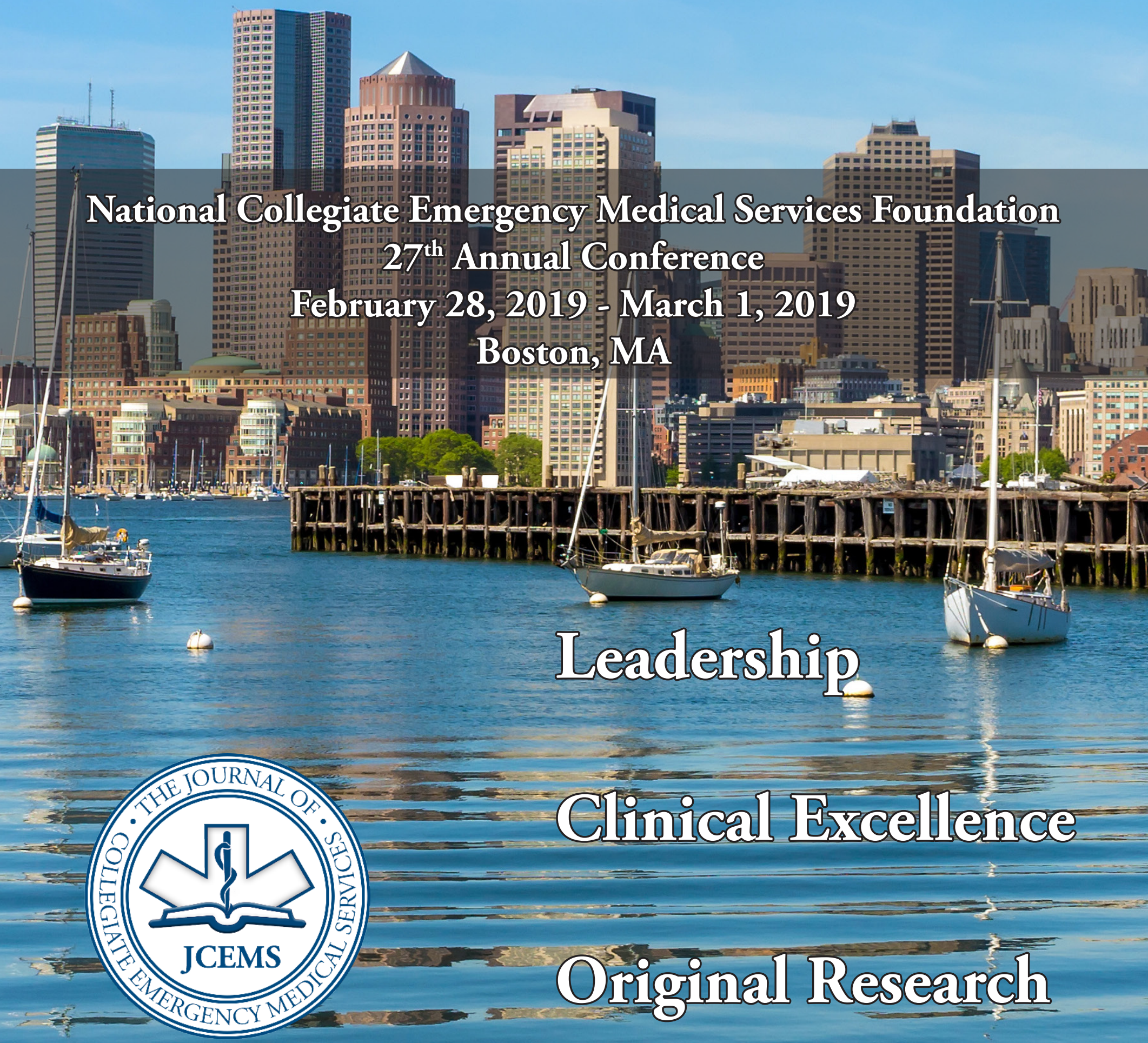

